\title{
RISK FACTORS \\ Long-term low mortality in patients without coronary artery calcium
}

Patients who have no detectable coronary artery calcium (CAC) and are in low or intermediate categories of cardiovascular risk have a 15-year prognostic warranty period for all-cause mortality of $<1 \%$. In these asymptomatic individuals without known coronary artery disease (CAD), $\mathrm{CAC}$ is a more reliable predictor of mortality than traditional risk factors.

$\mathrm{CAC}$ is a measure of subclinical CAD that is known to have prognostic value for cardiovascular disease. However, the association between CAC and traditional risk factors is far from perfect. In a study of 9,715 asymptomatic individuals who underwent CAC imaging by electron beam CT, all-cause mortality was assessed over a mean follow-up period of 14.6 years. The cohort was stratified separately by both the Framingham Risk Score (FRS) and National Cholesterol Education Program Adult Treatment Panel III (ATP III) categories, to compare their long-term prognostic values with that of CAC scores.
Of 936 deaths, 229 occurred in the group of 4,864 individuals with a CAC score of 0 . FRS stratification placed $50.0 \%$ of these individuals at low risk, $37.3 \%$ at intermediate risk, and $12.7 \%$ at high risk, whereas with ATP III these values were $62.9 \%, 23.7 \%$, and $13.4 \%$, respectively. By either stratification method, a CAC score of 0 and low-to-intermediate risk were associated with a 15 -year warranty period, wherein annual mortality remained $<1 \%$. By contrast, the warranty period for high cardiovascular risk was 5 years (FRS) or 6 years (ATP III).

With a CAC score $>0$, even individuals in the low-risk category had a maximum warranty period of only 5 years. Indeed, compared with $\mathrm{CAC}=0$ and low cardiovascular risk, the relative risk of mortality was higher with CAC $>0$ and low risk (HR 3.3 for FRS, HR 3.09 for ATP III) than $\mathrm{CAC}=0$ and high risk (HR 2.8 for FRS, HR 2.94 for ATP III). In multivariable analyses of this study population, CAC $>0$

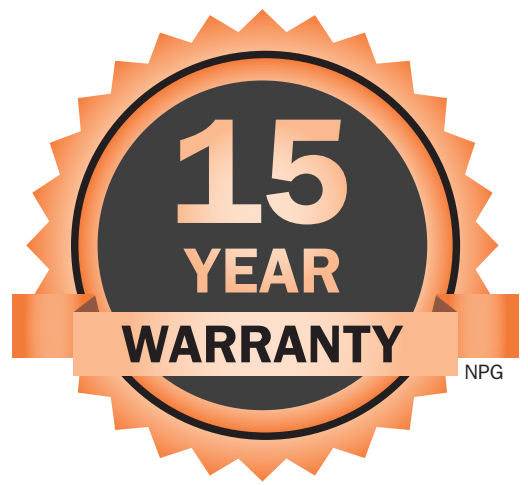

(compared with $\mathrm{CAC}=0$ ) was the strongest predictor of death (HR 2.67).

A CAC score of 0 results in a 'vascular age' that is lower than an individual's chronological age, and low vascular age corresponds to good long-term cardiovascular prognosis. Adding the CAC score to existing risk calculators can improve their performance, and might avoid unnecessary testing.

\section{Robert Phillips}

Original article Valenti, V. et al. A 15-year warranty period for asymptomatic individuals without coronary artery calcium. A prospective follow-up of 9,715 individuals. JACC Cardiovasc. Imaging doi:10.1016/j.jcmg.2015.01.025 